\title{
Quality Assessment of the Commonly Prescribed Antimicrobial Drug, Ciprofloxacin Tablets, Marketed in Tigray, Ethiopia
}

\author{
Getu Kahsay $^{1 *}$ and Awot G/Egziabher ${ }^{2}$ \\ $1^{*}$ Department of pharmaceutical chemistry, School of Pharmacy, College of Health Sciences, \\ Mekelle University, P.O.Box 1871, Mekelle, Ethiopia (*getu_kah@yahoo.com) \\ 2 Drug quality control and Toxicology laboratory, Ethiopian Drug Administration and Control \\ Authority, P.O.Box 5681, Addis Ababa, Ethiopia
}

\begin{abstract}
An attempt was made to assess the quality and compare the physicochemical equivalence of six brands of ciprofloxacin tablets marketed in Tigray, Ethiopia. Six brands of ciprofloxacin tablets were used in the study. Identity, weight uniformity test, disintegration test, dissolution test and assay for the content of active ingredients were performed using the methods described in the British Pharmacopoeia. All the samples passed the identity, disintegration, and dissolution tests but Ciflox failed to release $80 \%$ of the drug content within 30 minutes as stipulated in the pharmacopoeia. This product therefore does not comply with the BP 2004 dissolution tolerance limits. All of the brands examined pass the assay for content of active ingredient. This work revealed that the six brands included in the study complied with the physicochemical quality parameters except Ciflox which failed to meet the pharmacopoeial specification for dissolution test. Nevertheless the six brands showed that there is significant difference in the drug release in vitro $(p<0.05)$. To justify the specification limits of the in vitro dissolution test, an attempt should be made to establish a meaningful correlation between in vitro release characteristics and in vivo bioavailability parameters.
\end{abstract}

Keywords: Physicochemical properties; Quality; Bioequivalence; Dissolution rate; HPLC.

\section{INTRODUCTION}

The safety and efficacy of drug products can be guaranteed when their quality is reliable and reproducible from batch to batch. To ensure the requisite quality, drug manufacturers are required to test their products during and after manufacturing and at various intervals during the shelf life of the product (Chow, 1997). The quality of medicines is an integral part of access in light of ensuring that the pharmaceutical products are fit for their intended use, comply with the requirement of the marketing authorization and do not expose consumers to risks. To attain this objective there must be a system of quality assurance, which incorporates aspects including product development, manufacture, distribution, and storage. Many developing countries do not have an effective means of monitoring the quality of generic drug products in the market. This results in widespread distribution of substandard and/or counterfeit drug products. It was in view of this fact that the World Health Organization (WHO) issued guidelines for global standard and 
requirements for the registration, assessment, marketing, authorization, and quality control of generic pharmaceutical products (WHO, 1996; WHO, 2005).

The combination of market forces, the low per capita spending on pharmaceuticals by most of the population and the lack of adequate resources for controlling and monitoring the quality of drugs on the market creates an environment favorable for introducing low quality drugs in developing countries. Until very recently, the focus of developing country health advocates has been getting drugs into the supply chain at affordable prices. The quality of these drugs and the threat of counterfeit pharmaceuticals have been largely ignored. However, there is mounting evidence that counterfeit pharmaceuticals pose a serious threat to public health, especially in developing countries (Pecoul et al., 1999; WHO, 2005).

According to the United States Food and Drug Administration (FDA), bioavailability and bioequivalence of drug products, and drug product selection have emerged as critical issues in pharmacy and medicine during the last three decades. Concern about lowering health care costs has resulted in a tremendous increase in the use of generic drug products; currently about one half of all prescriptions written are for drugs that can be substituted with a generic product (Miller, 1990). In 1975, approximately 9\% of all prescription drugs dispensed were generic versions. This percentage rose to $20 \%$ in 1984 , and $40 \%$ in 1991 . Indeed substitution of generic drugs for brand name products is highly controversial and is often met with suspicion by health care providers and patients (Covington, 1992; Meredith, 2003). The FDA has mandated that all generic drugs meet the same standards as the brand name drugs in strength, safety, purity and performance. However, there are substandard and/or counterfeit generic drugs that don't satisfy the pharmacopoeial standards set for them.

In several developing countries, drug quality is a source of concern. There is a general feeling that there is a high incidence of drug preparations, which are not of acceptable quality (Ten Ham, 1992). Counterfeit pharmaceuticals comprise a significant and growing problem. Pharmaceutical counterfeiting is a pervasive problem, impacting nations of every size and income level and drugs of every description (Alubo, 1994; Graciela, 2001). There are many reports of the availability of counterfeit medicines, not only in developing countries but also in Europe and USA (Shakoor et al., 1997).

Reports indicate that there are substandard drugs circulating in the market in different developing countries. Marketing of such drugs has been widely reported in Africa, Asia, and Latin America. 
In 2003, WHO reported that fake drugs reported between 1999 and 2002 include analgesics and antipyretics (6\%), antimalarials (7\%), anti-asthma and anti-allergy (8\%), antibiotics (28\%), hormones and steroids (18\%) and other therapeutic categories (33\%). Thus, the aforementioned problems have resulted in a weak therapeutic efficiency, selection of resistance strains and poor quality of numerous drugs (Menkes, 1997; Newton et al., 2001; Taylor et al., 2001).

Lack of competent regulatory authorities and poor quality control practices in some countries, have allowed the availability of poor quality drugs. These could be the widespread counterfeiting of medicines, decomposition of the active ingredient in drug dosage form due to high temperature and humidity of the storage condition, and inadequate quality assurance systems during the manufacture of pharmaceutical products (Risha et al., 2003; Kaymba et al., 2004; Hebron et al., 2005).

Preliminary physicochemical assessment of drug products has a paramount importance in ensuring the quality of drug products. Generic drug products must satisfy the same standards of quality, efficacy and safety as those applicable to the innovator products. In vitro dissolution testing can be a valuable predictor of the in vivo bioavailability and bioequivalence of oral solid dosage forms (Itiola and Pilpel, 1996). Thus, the present study was carried out to evaluate the physicochemical quality and equivalence of six brands of ciprofloxacin tablets purchased from retail pharmacy outlets in Mekelle using in vitro methods. The preliminary study was aimed at obtaining baseline data towards the establishment of bioequivalence of the tablets thereby rendering information for regulatory bodies as well as manufacturers to ensure the quality of these essential drug products through monitoring by post-marketing surveillance.

\section{MATERIALS AND METHODS}

\subsection{Materials}

Table 1 shows the detailed information on ciprofloxacin tablet formulations included in the study. The drugs were selected on the basis of being included in the Ethiopian national drug list obtained from the Ethiopian Drug Administration and Control Authority (DACA). Ciprofloxacin tablets were obtained from the retail outlets mainly from private pharmacies and drug stores in Mekelle, Tigray. The drug samples were anonymously purchased in their original package as supplied by the manufacturers and protected from direct sunlight. 
Table 1. Detailed information on ciprofloxacin $500 \mathrm{mg}$ tablet formulations evaluated for quality.

Manufacturer Brand name StrengthBatch $N^{0}$ Mfg. Date Exp. Date

Sunshine Pharmaceuticals, Ethiopia $\quad$ Ciprofloxacin500 mg 07011201 Jul-2004 Jan-2009

\begin{tabular}{llll}
\hline Kwang Mayung Pharma, Korea & Floxine & $500 \mathrm{mg} 7008$ & Mar-2007 Mar-2010
\end{tabular}

Addis Pharmaceutical Factory S.C,

Ethiopia

Cadila Pharmaceuticals Ltd., India $\quad$ Ciprodac $500 \mathrm{mg}$ E6047 $\quad$ Oct-2006 Sep-2009

C.P.M Contract Pharma GmbH \& Co.

KG, Germany

Cipro-denk 500mg 1117

Jul-2007 Jul-2010

Torrent Pharmaceuticals Ltd, India $\quad$ Quintor 500 mg B3997017 Feb-2007 Jan-2011

\subsubsection{Instruments and equipment}

A high performance liquid chromatography (HPLC), a Pharma test dissolution tester, a UV/VIS spectrophotometer, an Ultrasonicator, an Electronic micro balance, and a high performance thin layer chromatography (HPTLC) aluminum sheets were used for the study.

\subsubsection{Chemicals and reagents}

The following chemicals and reagents were used in the study: Acetonitrile and ammonia HPLC grade, BDH (UK), dichloromethane, orthophosphoric acid, Methanol and Triethylamine, HPLC grade, were obtained from Sigma-Aldrich (Germany). Water, double distilled and deionized inhouse. The Ciprofloxacin $\mathrm{HCl}$ working standard, Lot Number XLM0725369 with purity or potency of 99.87\% was obtained from Aurobindo pharmaceutical industries (India).

\subsection{Methods}

Six brands of ciprofloxacin $500 \mathrm{mg}$ tablets were used in the study. Identity, weight uniformity, dissolution test and assay for the content of active ingredients were done as described in the British Pharmacopoeia (BP, 2004). All the assays were carried out in triplicate except the dissolution tests which were done six times as indicated in BP. Dissolution profiles were constructed for each drug product.

\subsubsection{Identification test}

The identity of the drug was confirmed by high performance thin layer chromatography (HPTLC) with silica gel 60F 254 pre-coated (10x20) cm obtained from Merck (UK). The mobile phase was consisted of a mixture of methanol, dichloromethane, ammonia and acetonitrile (40:40:20:10) as stated in BP 2004. A test solution of 0.05\% w/v was prepared by mixing $750 \mathrm{ml}$ of water with powdered tablets of ciprofloxacin equivalent to $2 \mathrm{gm}$ of ciprofloxacin in ultrasonic 
bath for 20 minutes and further diluted to $1000 \mathrm{ml}$. A standard solution of ciprofloxacin hydrochloride was prepared by dissolving $58 \mathrm{mg}$ with $100 \mathrm{ml}$ of the mobile phase. A third solution, which was a 1:1 mixture of the reference and test solution, was prepared. A $10 \mu \mathrm{l}$ of each of the solutions was applied separately as bands on pre-coated silica gel plate. The plates were placed in a suitable chromatographic chamber and the chromatogram was developed using the aforementioned solvent system. The plate was removed after the solvent has moved about three-fourth of the plate. The plate was dried in air for 15 minutes and examined under UV-light, $254 \mathrm{~nm}$. The distance of each spot from the point of origin was marked with a pencil, measured and recorded.

\subsubsection{Weight uniformity test}

The test for uniformity of weight for each brand of ciprofloxacin tablets was carried out as described in BP 2004. The weights of twenty tablets were determined individually using an electronic micro balance; model AAA 250L, Adams Co. (UK). The mean tablet weight and standard deviation were calculated.

\subsubsection{Disintegration time (DT) test}

Disintegration time test for the tablets was carried out according to the specification given in BP 2004. A $900 \mathrm{ml}$ beaker was filled with $37 \pm 0.5^{\circ} \mathrm{C}$ water. Six tablets were placed into the basketrack assembly and connected to the disintegration apparatus. The apparatus and the timer were started simultaneously and the time required for each tablet to disintegrate was recorded and the average time calculated.

\subsubsection{Dissolution}

Dissolution test was done according to BP 2004 specifications using dissolution apparatus type II (Paddle apparatus) obtained from Pharma test (Germany). Vessels consisting of $900 \mathrm{ml}$ of water as the dissolution medium were employed. The vessels were added to a circulating water bath maintained at $37 \pm 0.5^{\circ} \mathrm{C}$ and allowed to equilibrate. Once the desired temperature was attained, the tablets were added to the center bottom of the vessel and the paddle was rotated at $50 \mathrm{rpm} .10$ $\mathrm{ml}$ samples of the dissolution medium were withdrawn from each vessel using a syringe after 10 , 20, 30, 40, 50 and 60 minutes and an equivalent amount of the dissolution medium was immediately introduced as a replacement. The samples were filtered and suitably diluted with the dissolution medium. The concentrations were determined by measuring the absorbance at 276 nm using a UV/VIS spectrophotometer, Spectro genesis 5 (UK). Solution of ciprofloxacin 
reference standard was also prepared using the dissolution medium and absorbance was measured. Water was used as a blank. All the necessary corrections for dilution were made when calculating the percent of drug released and calibration curve for the dissolution was set.

\subsubsection{Assay of ciprofloxacin tablets}

All the eight drug products were assayed for the drug content according to the method outlined in the individual drug monographs of the British Pharmacopoeia (BP, 2004). The assay was carried out using HPLC. The HPLC system consisted of a solvent pump (model LC-10AT VP), an UVdetector (SPD-10A VP), a system controller (SCL-10A VP), a degasser (DGU-14A), a software (CLASS VP Version 5.02) all from Shimadzu Instruments (Japan). A stainless steel column of ODS (25 cm x $4.6 \mathrm{~mm}$ ) obtained from TEKNOKROMA, S. Coop. Company Ltd (Spain, Barcelona) was packed with stationary phase of $7 \mu \mathrm{m}$ particle size; UV-VIS detector at $278 \mathrm{~nm}$; mobile phase: a mixture of acetonitrile and phosphoric acid the $\mathrm{p}^{\mathrm{H}}$ of which was adjusted to 3.0 with triethylamine (TEA) in the ratio of 20:80 (v/v); flow rate of $2 \mathrm{ml} / \mathrm{min}$; injection volume: 10 $\mu \mathrm{l}$; and oven temperature: $40^{\circ} \mathrm{C}$. Twenty tablets were weighed and finely powdered. A quantity of the powdered tablets containing the equivalent of $0.5 \mathrm{gm}$ of ciprofloxacin was added to 250 $\mathrm{ml}$ of water. The solution was sonicated for 20 minutes and filtered. A portion of the filtrate (25 $\mathrm{ml}$ ) was withdrawn and diluted with sufficient amount of the mobile phase to give $100 \mathrm{ml}$ solution. $58 \mathrm{mg}$ of the reference standard was dissolved in $100 \mathrm{ml}$ of the mobile phase. The solutions were made ready on the sample vials and $10 \mu \mathrm{l}$ of each were injected to the system at intervals. The assays were repeated three times and the results presented were the mean of the three determinations. The content of ciprofloxacin was calculated from the peak areas of the chromatograms of the test and reference standard solutions and the declared content of ciprofloxacin in ciprofloxacin hydrochloride reference standard.

\subsubsection{Data Analysis}

Analytical data obtained from the experiments carried out were analyzed using ORIGIN ${ }^{\circledR}$ graphing and scientific analysis software program for manipulating and drawing of dissolution profiles; and GrahPad InStat Demo software program for statistical comparisons of dissolution profiles. 


\section{RESULTS AND DISCUSSION}

\subsection{Identification test of ciprofloxacin tablets}

The thin layer chromatographic (TLC) results for the identification of the drug samples are shown in table 2 . The retention factor $\left(\mathrm{R}_{\mathrm{f}}\right)$ of both the sample and the reference substance was calculated and compared. The TLC plates were examined under UV-light of $254 \mathrm{~nm}$. The results from table 2 revealed that the $R_{\mathrm{f}}$ values of the samples and the reference standard were almost the same. Thus, all the samples examined for the identity of the active ingredient passed the identification test as per the BP and USP/NF specifications.

\begin{tabular}{|lll|}
\hline $\begin{array}{l}\text { Table 2. Comparison of TLC retention factor }\left(\mathrm{R}_{\mathrm{f}} \text { values of }\right. \\
\text { different brands of ciprofloxacin tablets with a reference } \\
\text { standard. }\end{array}$ \\
\hline Brand name & $\boldsymbol{R}_{\boldsymbol{f}}$ (sample) & $\boldsymbol{R}_{\boldsymbol{f}}$ (standard) \\
\hline Ciprodac & 0.85 & 0.86 \\
\hline Ciprodenk & 0.86 & 0.86 \\
\hline Ciprofloxacin & 0.85 & 0.86 \\
\hline Quintor & 0.87 & 0.86 \\
\hline Floxine & 0.88 & 0.86 \\
\hline Ciflox & 0.86 & 0.86 \\
\hline
\end{tabular}

\subsection{Weight uniformity test}

The results of weight uniformity test are depicted in table 3 . The mean tablet weight, the number of tablets within and outside the BP specification limit range for the different products is included.

Weight uniformity test is required to assure that the drug content in each unit dose is distributed in a narrow range around the label strength. If the drug substance forms the greater part of the oral solid dosage form, any weight variation obviously reflects variation in the content of active ingredient. According to the specification outlined in BP 2004, the test for uniformity of weight for drug products where the strength is $>250 \mathrm{mg}$ (film coated tablets), a $\pm 5 \%$ of the average pass the test for uniformity of weight. The results, thus, indicate that six of the products possess acceptable uniformity of weight as per the pharmacopoeia limit. Moreover, the relative standard deviation of the products is less than 6 and the number of tablets outside the BP range is within the limit stipulated in the pharmacopoeia. 


\begin{tabular}{|c|c|c|c|c|}
\hline Brand name & $\begin{array}{l}\text { Mean }(\mathrm{mg}) \text { of } 20 \\
\text { determinations }\end{array}$ & $\pm R S D(\%)$ & $\begin{array}{l}N \text { o. of tablets within } \\
\text { the BP range }\end{array}$ & $\begin{array}{l}N \text { o. of tablets outside } \\
\text { the BP range }\end{array}$ \\
\hline Ciprodac & 752.50 & 3.33 & 18 & 2 \\
\hline Ciprodenk & 732.20 & 0.83 & 20 & 0 \\
\hline Ciprofloxacin & 760.80 & 5.34 & 19 & 1 \\
\hline Quintor & 749.51 & 0.50 & 20 & 0 \\
\hline Floxine & 738.05 & 0.95 & 20 & 0 \\
\hline Ciflox & 685.60 & 1.02 & 20 & 0 \\
\hline
\end{tabular}

\subsection{Disintegration test}

The mean disintegration times of the different brands of the ciprofloxacin tablets included in the study is shown in table 4. As indicated in the table, the mean disintegration time for all the products was within the pharmacopoeia limit. For a drug to be absorbed from a solid dosage form after oral administration, it must first be in solution, and the first important step toward this condition is usually the break-up of the tablet; a process known as disintegration. According to the BP 2004, the time limit for disintegration of film coated tablets is $<30 \mathrm{~min}$. All the products comply with the outlined specification time for disintegration of film coated tablets. Moreover all the drug products had a mean disintegration time of less than five minutes.

\begin{tabular}{|lll|}
\hline Table 4. Disintegration time (DT) of randomly selected ciprofloxacin 500 mg tablets (n=6). \\
\hline Brand name & Mean (min) of six determinations & $\mathbf{\pm R S D ( \% )}$ \\
\hline Ciprodac & 3.00 & 0.22 \\
\hline Ciprodenk & 2.00 & 0.10 \\
\hline Ciprofloxacin & 4.00 & 0.00 \\
\hline Quintor & 3.00 & 3.16 \\
\hline Floxine & 4.00 & 0.40 \\
\hline Ciflox & 4.00 & 1.00 \\
\hline
\end{tabular}

\subsection{Assay of ciprofloxacin tablets}

The results for the mean percentage label claim of the different brands of ciprofloxacin $500 \mathrm{mg}$ tablets included in the study are depicted in table 5. The products were assayed according to the method outlined in BP, 2004. In this, it is described that ciprofloxacin tablets should contain not less than $95.0 \%$ and not more than $105.0 \%$ of the stated amount. As indicated in table 5, all brands of ciprofloxacin tablets passed as per the BP specification. The highest percentage content was obtained for Ciprodac (105.78) \%, while the least drug content was obtained for Ciflox 
(98.70) \%. Statistical comparison for drug content indicates that with 95\% confidence interval, there is exists significant difference in the drug content among the different brands $(\mathrm{P}<0.05)$.

\subsection{Dissolution test}

The dissolution profile of the six brands is illustrated in figure 1 and their chromatograms are indicated in figures 2, 3 and 4; and for reference standards in figure 5. Products with different formulations, different inactive ingredients, and different formulation design may have different dissolution profiles or release characteristics and therefore may have different bioavailability. In the present study, the dissolution profiles of the six products were tested according to the method described in the BP, 2004. It is stated that the amount of ciprofloxacin released within 30 minutes is not less than $80 \%$ of the stated amount.

\begin{tabular}{|lll|}
\hline \multicolumn{3}{|l|}{ Table 5. The percentage drug content of randomly selected ciprofloxacin $500 \mathrm{mg}$ tablets (n=20). } \\
\hline Brand name & $\begin{array}{l}\text { Mean percentage label claim (Mean of } \\
\text { triplicate determinations) }\end{array}$ & 0.085 \\
\hline Ciprodac & 105.78 & 0.048 \\
\hline Ciprodenk & 101.95 & 0.056 \\
\hline Ciprofloxacin & 100.23 & 0.101 \\
\hline Quintor & 105.65 & 0.102 \\
\hline Floxine & 102.51 & 0.102 \\
\hline Ciflox & 98.700 & \\
\hline
\end{tabular}

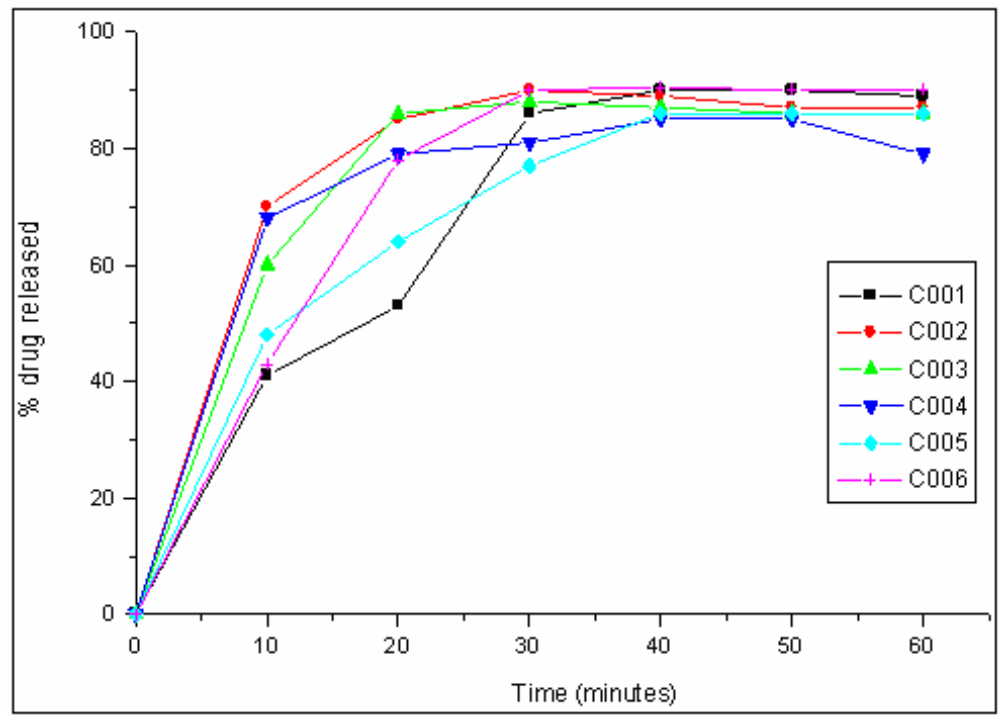

Figure 1. Dissolution profiles of the different brands of ciprofloxacin tablets in water medium.

All the brands of ciprofloxacin tablets studied released more than $80 \%$ within 30 minutes except Ciflox which has released only $77.60( \pm 2.84)$. Hence, five of the products complied with the BP 
and USP dissolution tolerance limits i.e. not less than $80 \%$ of the drug is released in 30 minutes but Ciflox failed to release the stated amount. It was observed that ciprodenk with the smallest disintegration time (2 $\mathrm{min}$ ) had a mean percentage drug release of $90.31 \%$ within 30 minutes- the maximum of all the brands investigated. This is in line with the general concept that fast disintegrating tablets release their drug substance easily and vice versa.

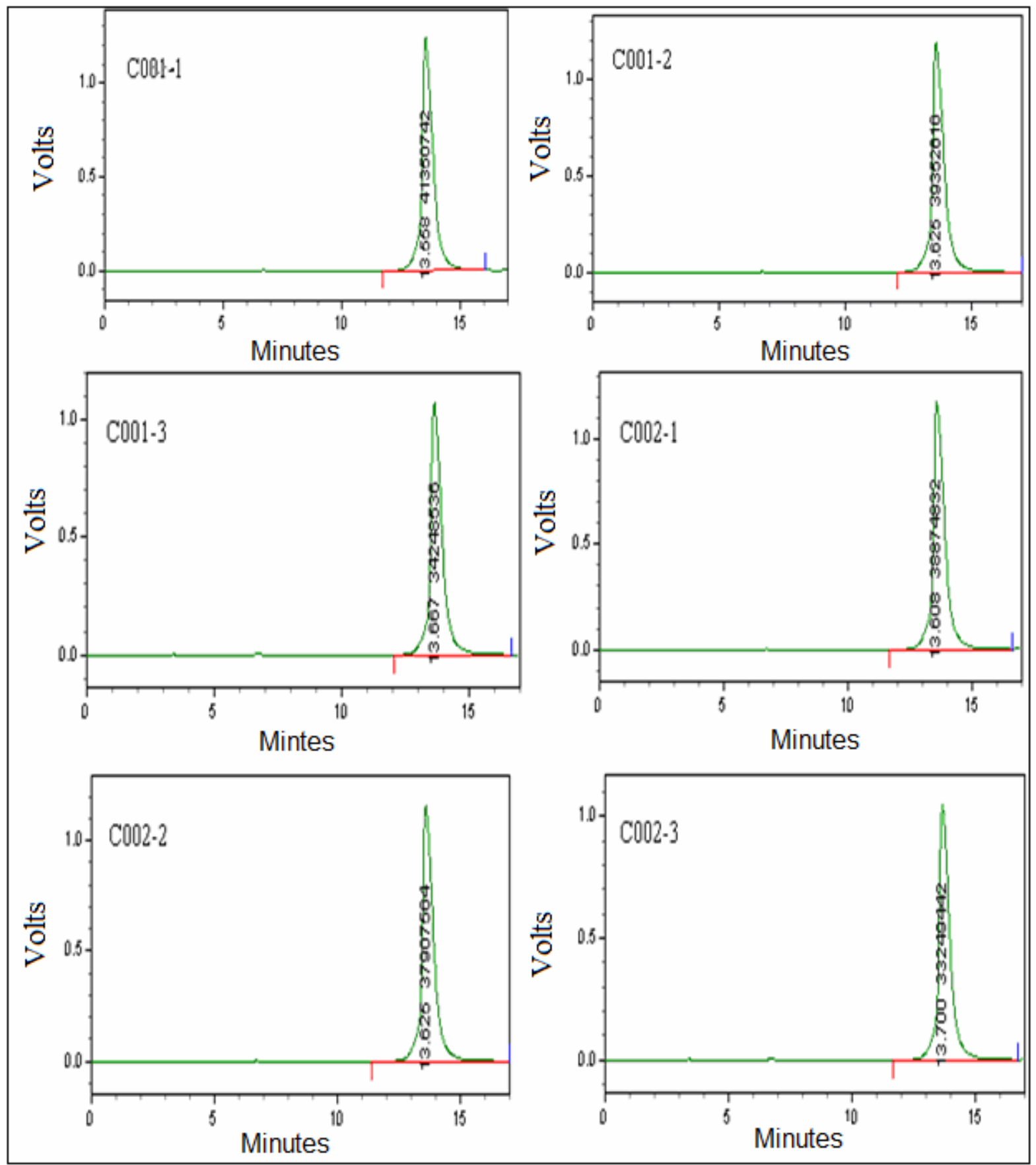

Figure 2. HPLC chromatograms of the two brands of ciprofloxacin tablets with sample codes C001 and C002. 
In general, the six brands showed differences in their drug release in vitro which could also result in differences in their bioavailability in vivo. However, in vitro testing only predicts the in vivo bioavailability and bioequivalence of oral solid dosage forms. It does not exclusively indicate the in vivo performance of a drug. The value of dissolution as a quality control tool for predicting in vivo performance of a drug product is significantly enhanced if an in vitro-in vivo relationship (correlation or association) is established (FDA, 1997; Sathe et al., 1997, 2001).

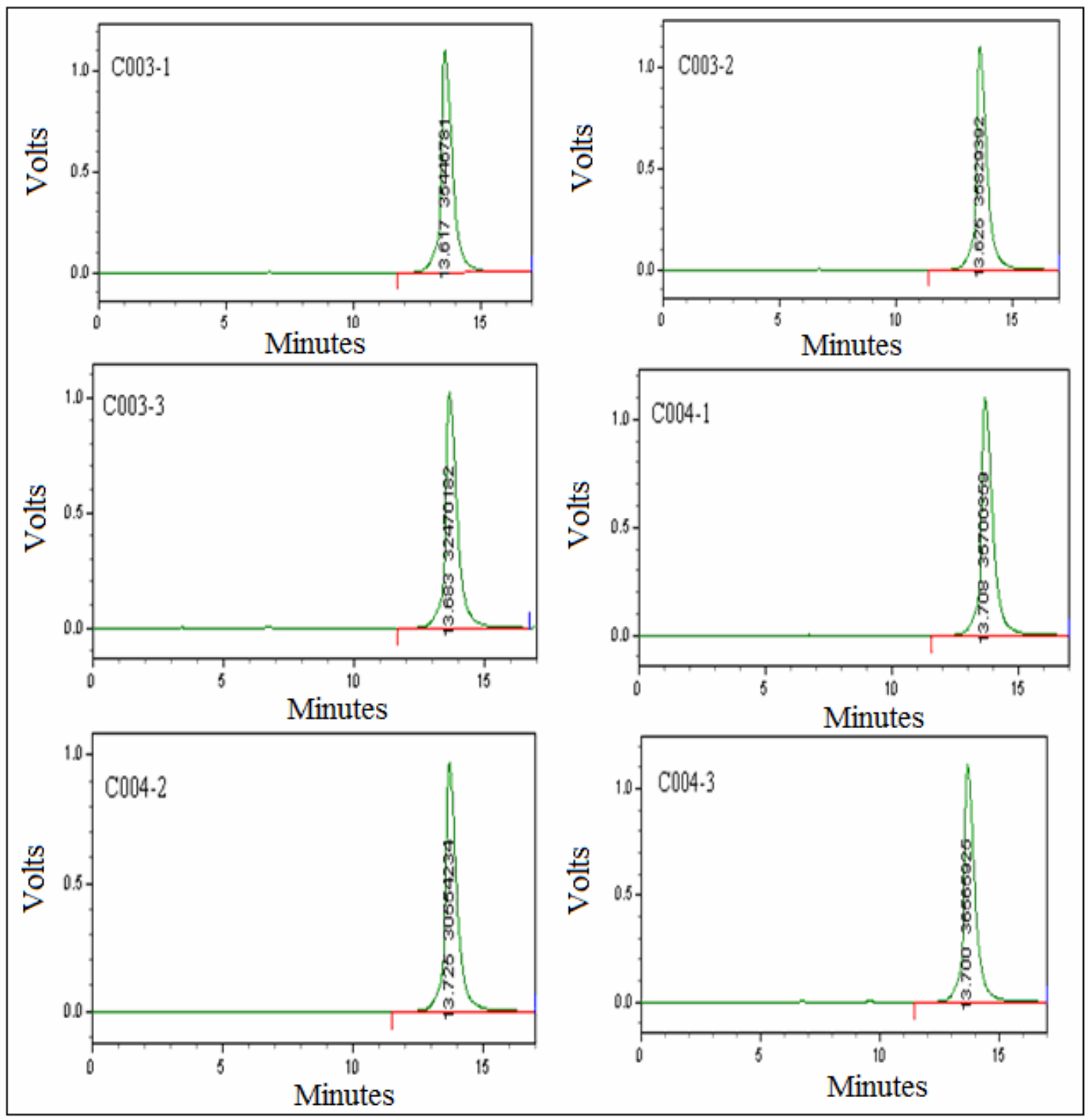

Figure 3. HPLC chromatograms of the two brands of ciprofloxacin tablets with sample codes C003 and C004. 


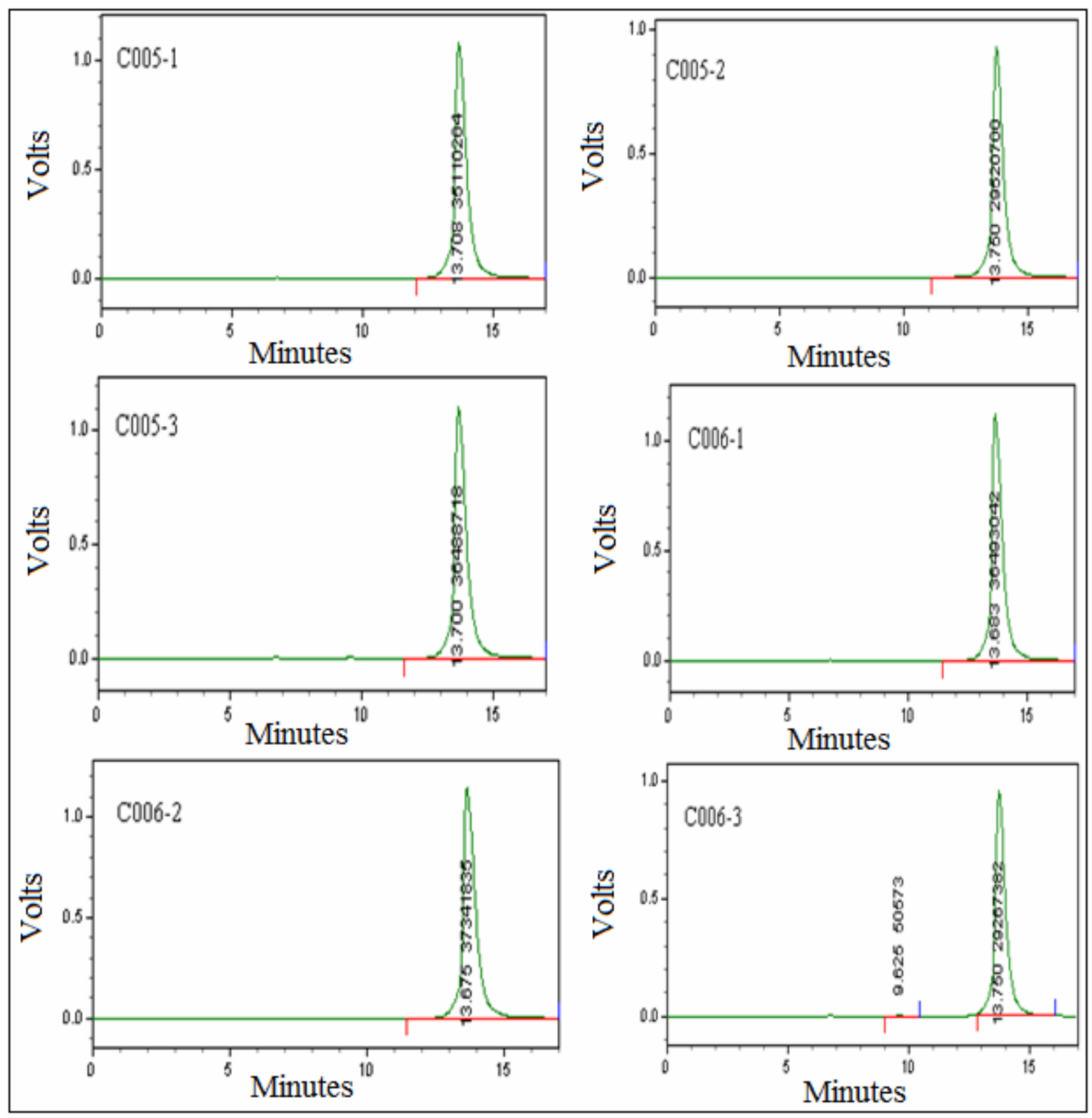

Figure 4. HPLC chromatograms of the two brands of ciprofloxacin tablets with sample codes C005 and C006. 


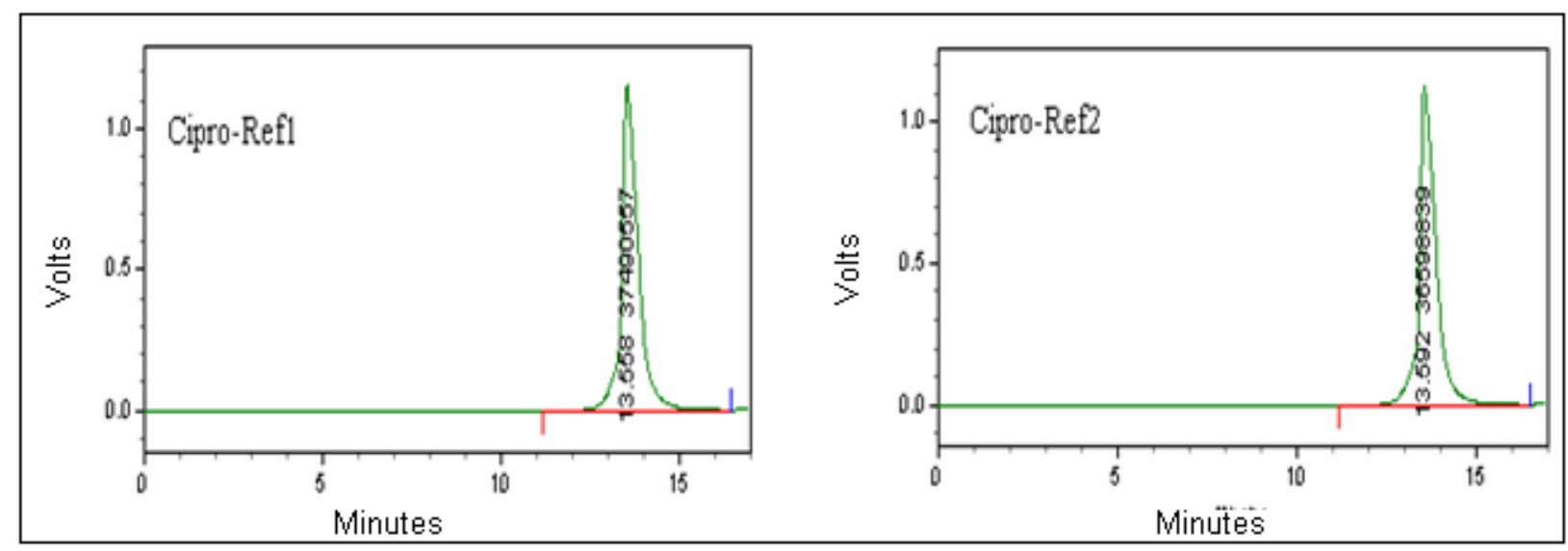

Figure 5. HPLC chromatograms of the reference standard.

\section{CONCLUSION}

The study attempted to assess the quality of ciprofloxacin tablets and the physicochemical equivalence of the six brands. The physicochemical evaluation showed that all of the ciprofloxacin tablets tested for identity of the active ingredient, weight uniformity, and disintegration time complied with the pharmacopoeial specifications described in the BP and USP/NF. Moreover, the ciprofloxacin tablets included in the study have mean drug content within the compendial tolerance limits. Statistical comparison for drug content indicates that with 95\% confidence interval, there is no significant difference among the brands $(\mathrm{P}<0.05)$. Five brands complied with the BP and USP dissolution tolerance limits but Ciflox failed to release the stated amount. In addition, the six brands showed that there is significant difference in the drug release in vitro $(p<0.05)$. To justify the specification limits of the in vitro dissolution test, further study should be made to establish a meaningful correlation between in vitro release characteristics and in vivo bioavailability parameters. Hence, the safety, quality and efficacy of essential drugs in the region, should be continuously monitored through post marketing surveillance practices.

\section{ACKNOWLEDGEMENTS}

The Authors would like to thank Mekelle University for the financial support and the Ethiopian Drug Administration and Control Authority for providing laboratory facility for this research work. 


\section{REFERENCES}

Alubo, S.O. 1994. Death for Sale: A study of drug poisoning and deaths in Nigeria. Soc. Sci. Med., 38: 97-103.

British Pharmacopoeia B. P, 2004. Her Majesty’s Stationery Office, London, Vol. III.

Chow, S. 1997. Pharmaceutical Validation and Process Controls in Drug Development. J. Drug Information., 31: 1195-1201.

Covington, T.R. 1992. Generic drug utilization: Overview and guidelines for product use. Clin. Res. Reg. Affairs, 9: 103-126.

FDA, 1997. Center for Drug Evaluation and Research (CDER): Guidance for Industry: Dissolution Testing of Immediate Release Solid Oral Dosage Forms, pp. 6-8.

Graciela, I.R. 2001. Argentina to Crack Down on pharmaceutical Black market. The Lancet, 358: 47.

Hebron, Y., Tetteyy, J.A., Pournamdari, M \& Watson, D.G. 2005. The chemical and pharmaceutical equivalence of sulphadoxine/ pyrimethamine tablets sold on the Tanzanian market. J. Clin. Pharm. Ther., 30:575-581.

Itiola, O.A \& Pilpel, N. 1996. Effects of interacting variables on the disintegration and dissolution of metronidazole tablets. Pharmazie, 51: 987-989.

Kaymba, P.C., Risha, P.G \& Shewiyo, D. 2004. The quality of essential antimicrobial and antimalarial drugs marketed in Rwanda and Tanzania: Influence of tropical storage conditions on in vitro dissolution. J. Clin. Pharm. Ther., 29: 331-338.

Menkes, D.B. 1997. Hazardous drugs in developing countries. The market may be healthier than the people. British Med. J., 315: 1557.

Meredith, P. 2003. Bioequivalence and other unresolved issues in generic drug substitution. Clin. Ther., 25: 2875-2890.

Miller, SW \& Strom, JG. 1990. Drug Product Selection: Implications for the Geriatric Patient. The Consultant Pharmacist, 5: 30-37.

Newton, P., Proux, S., Green, M., Smithuis, F., Rozendaal, J., Prakongpan, S., Chotivanich, K., Mayxay, M., Looareesuwan, S., Farrar, J., Nosten, F \& White, N.J. 2001. Fake artesunate in Southeast Asia. The Lancet, 357: 1948-1950.

Pecoul, B., Chirac, P., Trouiller, P \& Pinel, J. 1999. Access to essential drugs in poor countries: A lost battle? Am. J. Med. Ass., 281: 361-367. 
Risha, P.G., Vervaet, C., Vergote, G., Bortel, L.V \& Remon, J.P. 2003. Drug formulations intended for the global market should be tested for stability under tropical climatic conditions. European J. Clinical Pharmacology, 59: 135-141.

Sathe, P., Tsong, Y \& Shah, V.P. 1997. In Vitro dissolution profile comparison and in vitro in vivo relation: Carbamazepine case. Adv. Exp. Med. Biol., 432: 31-42.

Sathe, P., Tsong, Y \& Shah, V.P. 2001. In Vitro dissolution profile comparison and in vitro in vivo relation: Carbamazepine case. Pharm. Res., 18: 1729-1734.

Shakoor, O., Taylor, R.B \& Berhens, R.H. 1997. Assessment of the incidence of substandard drugs in developing countries. Trop. Med. Int. Health., 2: 839-845.

Taylor, R.B., Shakoor, O \& Behrens, R.H. 2001. Pharmacopoeial quality of drugs supplied by Nigerian pharmacies. The Lancet, 357:1933.

Ten Ham, M. 1992. Counterfeit drugs: Implications for health. Adverse Drug Reactions and Toxicology Reviews II, pp. 59-65.

WHO, 1996. Technical Report Series No. 863. Expert Committee on specifications for Pharmaceutical Preparations. $34^{\text {th }}$ Report World Health Organization, Geneva.

WHO, 2005. Regional strategy for improving access to essential medicines in the Western Pacific Region 2005-2010. World Health Organization, Geneva. 